\title{
RENDIMENTO DO DESDOBRO DE MADEIRA EM SERRARIA CONVENCIONAL E DIFERENTES SIMULAÇÕES UTILIZANDO OTIMIZADOR COMPUTACIONAL
}

\author{
YIELD OF SAWING IN CONVENTIONAL SAWMILL AND DIFFERENT SIMULATIONS USING \\ COMPUTATIONAL OPTIMIZER
}

\author{
Edson Luis Serpe ${ }^{1}$, Afonso Figueiredo Filho ${ }^{2}$, Julio Eduardo Arce \\ ${ }^{1}$ LWARCEL, Lençóis Paulista, Paraná, Brasil-serpe.edson@gmail.com \\ ${ }^{2}$ Universidade Estadual do Centro Oeste, Irati, Paraná, Brasil - afigfilho@gmail.com \\ ${ }^{3}$ Universidade Federal do Paraná, Curitiba, Paraná, Brasil-jarce@ufpr.br
}

RESUMO

\begin{abstract}
O rendimento e a análise econômica do desdobro da madeira serrada de Pinus spp., em serraria convencional no município de General Carneiro, região sul do estado do Paraná, foram estudados utilizando a metodologia empregada pela serraria (método convencional) e simulando diferentes opções otimizadas de produtos, no software otimizador MaxiTora. Com objetivo de determinar o rendimento do desdobro e análise econômica para desdobro não programado e programado, para diferentes classes de diâmetro de toras, foram separadas em dois lotes, toras de classe tipo 1 (diâmetros acima de $23 \mathrm{~cm}$ ) e toras de classe tipo 2 (diâmetro de 16 a 22,99 cm). A industrialização das toras foi realizada com cortes paralelos (live saw), sendo o desdobro principal realizado em serra fita e para o ajuste da largura das peças foi utilizada a serra circular. O rendimento do desdobro de toras não programado proporciona rendimento de 35,79 a 45,09\%, contudo o rendimento do desdobro de toras considerando a utilização de software de otimização de toras varia de 36,81 a 55,15\%. O maior benefício econômico de madeira serrada é produzido em toras de 16 a $23 \mathrm{~cm}$ de diâmetro.
\end{abstract}

Palavras-chave: Controle de Qualidade, Industrialização, Multiprodutos, Software, Toras.

\begin{abstract}
The yield and economic analysis of the sawing lumber of Pinus spp. in a conventional sawmill in the municipality of General Carneiro, southern region of the state of Paraná, were studied using the methodology used by the sawmill (conventional method) and simulating different options of the products in the MaxiTora optimizer software. In order to determine the sawnmill yield and economic analysis for sawnmill non-programed and programmed sawnmill, were separed in two lotes for different classes of log diameter, logs of class 1 (diameter above $23 \mathrm{~cm}$ ) and logs of type 2 (diameter 16 to $22.99 \mathrm{~cm}$ ). The industrialization of the logs was carried out with parallel cuts (live saw), the main sawing was carried out in ribbon saw and for the adjustment of the width of the pieces the circular saw was used. The unprogrammed wood logs sawnmill yields is 35.79 to $45.09 \%$, however the sawnmill considering the use of optimization software yields from 36.81 to $55.15 \%$. The greatest economic benefit of sawn wood is produced in logs of 16 to $23 \mathrm{~cm}$ in diameter.
\end{abstract}

Keywords: Quality Control, Industrialization, Multiproducts, Software, Wood logs. 


\section{INTRODUÇÃO}

O desdobro é o processo de redução da tora inteira, por meio do corte longitudinal, em peças menores de seç̧ão retangular ou quadrada geralmente realizado em serrarias (FAGUNDES, 2003).

A metodologia de desdobro utilizada determina o maior ou menor rendimento da matéria-prima. A determinação dos rendimentos obtidos numa serraria, para as mais variadas classes diamétricas e produtos, é fator importante economicamente (MURARA JUNIOR et al., 2013).

A metodologia de desdobro de toras pode ser realizada de duas maneiras: convencional e programada. No convencional na maioria das vezes o sistema de corte tangencial é utilizado tentando obter o maior número de peças. Já a programada busca um sistema de corte previamente examinado, objetivando a máxima utilização da tora (LEITE, 1994).

O desdobro de madeira com cortes tangenciais, também conhecido como cortes paralelos (live saw), é o mais utilizado no Brasil, principalmente nas serrarias de pequeno porte. Neste método os cortes são realizados em uma mesma direção, utilizando uma repetição de passagens. Apesar da facilidade e da precisão, este método pode apresentar grande perda de madeira, reduzindo o rendimento (SILVA, 2001).

A simulação de sistemas pode ser utilizada no planejamento da produção de serrarias, na busca de melhorias de processos, contribuindo para a competitividade das empresas no mercado (HEINRICH, 2010). Essa é uma relevante ferramenta para o apoio à tomada de decisão, estando presente nas organizações em diversos campos de atuação (HOLLOCKS, 2006).

O MaxiTora, é um software desenvolvido para uso na indústria de processamento de madeira serrada. Esta ferramenta computacional auxilia na definição do padrão de corte possibilitando maior precisão, uma visualização prévia de produtos e rendimentos obtidos para as várias classes diamétricas processadas (NOVAK, 2007).

É um software de simulação de desdobro, onde por meio de uma linha de produtos ou uma determinada necessidade de produtos, é possível definir a classe diamétrica mais apropriada, em termos de rendimento (OPTIMBER, 2016).

O trabalho objetivou avaliar o rendimento do desdobro de madeira serrada de Pinus spp. em serraria convencional e simulando diferentes produtos em duas diferentes classes de diâmetro utilizando o software Maxi tora.

\section{MATERIAL E MÉTODOS}

\section{Características da serraria}

A transformação das toras em madeira serrada, foi realizada em uma serraria de desdobro de toras de Pinus spp. localizada no município de General Carneiro, estado do Paraná, a qual representa o perfil das indústrias deste setor na região.

\section{Coleta dos dados}

Para a industrialização foram utilizadas 478 toras, as quais foram separadas por classes de diâmetro "Serraria 1" e "Serraria 2" (Tabela 1).

Tabela 1. Sortimentos e número de toras utilizadas para industrialização

\begin{tabular}{cccc}
\hline Classe & $\begin{array}{c}\text { Diâmetro } \\
(\mathbf{c m})\end{array}$ & $\begin{array}{c}\text { Comprimento } \\
(\mathbf{m})\end{array}$ & $\begin{array}{c}\text { Número de } \\
\text { toras }\end{array}$ \\
\hline Serraria 1 & {$[>23)$} & 2,65 & 176 \\
Serraria 2 & {$[16-23)$} & 2,65 & 302 \\
\hline
\end{tabular}

O volume das toras foi calculado pela fórmula de Smalian, e o custo da matéria-prima foi calculado multiplicando o volume das toras por classe e o preço de madeira carregada no caminhão praticados pela serraria (Tabela 2).

$$
\mathrm{Vt}=\frac{\left(\frac{\mathrm{dpf}^{2} x \pi}{4}+\frac{\mathrm{dpg}^{2} x \pi}{4}\right)}{2} \times \mathrm{l}
$$

Em que: $V t=$ Volume da tora $\left(\mathrm{m}^{3}\right) ; d p f=$ diâmetro da tora na ponta fina $(\mathrm{m})$; $d p g=$ diâmetro da tora na ponta grossa $(\mathrm{m})$; e I = comprimento do produto.

$$
\mathrm{Cmp}=\sum \mathrm{Vt} \times \mathrm{P}
$$

Em que: $\mathrm{Cmp}=$ Custo da matéria-prima; $\Sigma \mathrm{Vt}=$ Somatório do volume das toras da classe $\left(\mathrm{m}^{3}\right)$; e $\mathrm{P}=$ preço da tora.

Tabela 2. Preço de toras carregada no caminhão

\begin{tabular}{ccc}
\hline Classe & Classe de diâmetro $(\mathbf{c m})$ & $\mathbf{R} \$ \mathbf{m}^{\mathbf{3}}$ \\
\hline Serraria 1 & {$[>23)$} & 122,40 \\
Serraria 2 & {$[16-23)$} & 73,69 \\
\hline
\end{tabular}

A operação de desdobro foi realizada em serraria 
convencional, com cortes tangenciais, também conhecido como cortes paralelos (live saw).

$O$ desdobro principal foi realizado em serra fita, com lâmina de $2 \mathrm{~mm}$ de espessura e para o ajuste da largura das peças foi utilizada a serra circular com lâmina de $4 \mathrm{~mm}$.

Para o desdobro utilizou-se a metodologia empregada pela serraria, em que as toras são classificadas por sortimento e o processamento é definido pelo operador (método não programado), o qual verifica a melhor opção entre os produtos, buscando atingir o melhor rendimento.

Para a industrialização, as toras passaram pela primeira etapa de fabricação, sendo serradas retirando as costaneiras e definindo a espessura da peça (1, 2 ou 3 pol). Posteriormente passando pela segunda etapa, para dimensionar a largura da peça (1 a 12 pol).

Para o cálculo do volume em madeira serrada, os produtos obtidos em cada classe diamétrica foram medidos individualmente. O volume de cada produto foi determinado pelas seguintes equações:

$$
\mathrm{Vp}=\mathrm{E} \times \mathrm{L} \times \mathrm{C}
$$

Em que: $V p=$ volume da peça $\left(\mathrm{m}^{3}\right) ; E=$ espessura $d a$ peça $(\mathrm{m}) ; \mathrm{L}=$ largura da peça $(\mathrm{m})$; e $\mathrm{C}=$ comprimento da peça.

$$
\mathrm{Vpr}=\sum \mathrm{Vp}
$$

Em que: $\mathrm{Vpr}=$ volume total do produto $\left(\mathrm{m}^{3}\right)$; e $\sum \mathrm{Vt}=$ Somatório do volume das peças.

O rendimento volumétrico por classe de diâmetro, foi determinado utilizando a relação entre o volume total dos produtos e o volume total gerado das respectivas toras, com a seguinte equação (ROCHA, 2000).

$$
\mathrm{R} \%=\frac{\sum \mathrm{Vp}}{\sum \mathrm{Vt}} \times 100
$$

Em que: $\mathrm{R} \%$ = Rendimento em madeira serrada; $\Sigma \mathrm{Vp}=$ Somatório do volume dos produtos $\left(\mathrm{m}^{3}\right)$; e $\sum \mathrm{Vt}=$ Somatório dos volumes de toras $\left(\mathrm{m}^{3}\right)$.

\section{Simulação de otimização no desdobro utilizando o software MaxiTora}

A simulação de otimização no desdobro, foi realizada com o software MaxiTora. Na tela de cadastro, foram cadastrados os 32 produtos (Tabela 3 ), os quais se constituem como fonte de dados para a definição dos

\begin{tabular}{|c|c|c|c|}
\hline \multirow[b]{2}{*}{ Simulação } & \multicolumn{3}{|c|}{ Produto } \\
\hline & $\begin{array}{c}\text { 1" x 2" a 1" } \\
\text { x 12" }\end{array}$ & $\begin{array}{c}\text { 2" x 2" a 2" } \\
\text { x 12" }\end{array}$ & $\begin{array}{c}\text { 3" x 3" a 3" } \\
\text { x 12" }\end{array}$ \\
\hline MS & () & ( ) & $\Theta$ \\
\hline A & ○ & $\otimes$ & $\otimes$ \\
\hline B & $\otimes$ & ( & $\otimes$ \\
\hline C & $\otimes$ & $\otimes$ & () \\
\hline D & (-) & (-) & $\otimes$ \\
\hline$E$ & () & $\otimes$ & () \\
\hline $\mathrm{F}$ & $\otimes$ & $\Theta$ & $\Theta$ \\
\hline G & ( & $\Theta$ & ( \\
\hline
\end{tabular}
diagramas de corte específicos para as simulações.
Tabela 3. Lista de produtos de madeira serrada

": Polegada = 2,54 cm; MS: Madeira serrada no padrão da serraria; $\oslash$ : Produtos simulados; $\circledast$ : Produtos não simulados.

Fonte: Madsul (2015).

Os produtos foram cadastrados com o código de identificação (ID), nomeados e especificados com a "espessura" e "largura" do produto (Ex. 25 x $50 \mathrm{~mm}$ ), utilizando o método de processamento "Livre". Foram definidas as espessuras da serra vertical $(2 \mathrm{~mm})$ e horizontal ( $4 \mathrm{~mm}$ ), e otimização por volume.

Na tela de otimização de desdobro, foi selecionado a otimização livre, buscando entre os produtos cadastrados, definir o diagrama que proporcionar o melhor rendimento.

Para a comparação dos rendimentos gerados na indústria com diferentes simulações, foram definidos os comparativos apresentados na Tabela 3.

Para a análise estatística foi utilizado o delineamento inteiramente casualizado (DIC) com diferentes números de repetições, aplicado o teste de Bartlett, a análise de variância (ANOVA) teste de Tukey, ao nível de $5 \%$ de significância.

A maximização da receita bruta da madeira serrada, foi analisada realizando o comparativo entre a receita bruta da madeira serrada por sortimento de toras e a receita bruta simulada otimizada. Sendo considerando os valores de madeira serrada praticados pela serraria (Tabela 4).

A análise econômica foi análoga à descrita por Dobner Júnior et al. (2012) considerando o benefício econômico. A receita parcial da madeira serrada (receita bruta subtraída do valor de aquisição das toras) é dividida pelo valor de aquisição das toras, não sendo considerados os demais custos de produção. 
Tabela 4. Preços de madeira serrada

\begin{tabular}{|c|c|c|c|}
\hline \multirow{2}{*}{ Largura } & \multicolumn{3}{|c|}{ Espessura } \\
\hline & 1" & 2" & 3" \\
\hline 2" & $\mathrm{R} \$ 480,00$ & $\mathrm{R} \$ 550,00$ & - \\
\hline 3" & $\mathrm{R} \$ 480,00$ & $\mathrm{R} \$ 550,00$ & $\mathrm{R} \$ 550,00$ \\
\hline $4 "$ & $\mathrm{R} \$ 550,00$ & $R \$ 550,00$ & $\mathrm{R} \$ 550,00$ \\
\hline $5 "$ & $\mathrm{R} \$ 550,00$ & $\mathrm{R} \$ 550,00$ & $\mathrm{R} \$ 550,00$ \\
\hline 6" & $\mathrm{R} \$ 550,00$ & $\mathrm{R} \$ 550,00$ & $\mathrm{R} \$ 550,00$ \\
\hline 7" & $R \$ 550,00$ & $\mathrm{R} \$ 550,00$ & $\mathrm{R} \$ 550,00$ \\
\hline $8 \prime$ & $\mathrm{R} \$ 6^{2} 30,00$ & $\mathrm{R} \$ 630,00$ & $\mathrm{R} \$ 630,00$ \\
\hline $9 "$ & $\mathrm{R} \$ 630,00$ & $\mathrm{R} \$ 630,00$ & $\mathrm{R} \$ 630,00$ \\
\hline $10^{\prime \prime}$ & $\mathrm{R} \$ 630,00$ & $\mathrm{R} \$ 630,00$ & $\mathrm{R} \$ 630,00$ \\
\hline $11 "$ & $R \$ 630,00$ & $\mathrm{R} \$ 630,00$ & $\mathrm{R} \$ 630,00$ \\
\hline $12 "$ & $\mathrm{R} \$ 630,00$ & $\mathrm{R} \$ 630,00$ & $\mathrm{R} \$ 630,00$ \\
\hline
\end{tabular}

\section{RESULTADOS E DISCUSSÃO}

\section{Rendimento de madeira serrada}

O volume de toras utilizado para o desdobro foi de 33,06 $\mathrm{m}^{3}$ (176 toras) e de 25,42 $\mathrm{m}^{3}$ (302 toras), respectivamente para o sortimento "Serraria 1 " e "Serraria $2^{\prime \prime}$.

O rendimento da madeira serrada, pelo sistema de desdobro convencional e simulações otimizadas, buscando a maximização da receita bruta são apresentadas na Tabela 5.

Tabela 5. Rendimento de madeira serrada

\begin{tabular}{ccc}
\hline \multirow{2}{*}{ Simulação } & \multicolumn{2}{c}{ Rendimento (\%) } \\
\cline { 2 - 3 } & Serraria 1 & Serraria 2 \\
\hline MS & 45,09 & 35,79 \\
A & $53,82 a$ & $53,71 a$ \\
B & $47,24 a$ & $44,87 a$ \\
C & $41,68 a$ & $36,81 a$ \\
D & $54,04 a$ & $55,13 a$ \\
E & $53,91 a$ & $55,13 a$ \\
F & $49,00 a$ & $48,50 a$ \\
G & $54,04 a$ & $55,15 a$ \\
\hline
\end{tabular}

Médias seguidas da mesma letra não diferem estatisticamente pelo teste de Tukey ao considerar o nível de $5 \%$ de significância.

A madeira serrada considerando a opção de processamento livre, ou seja, sem a otimização, a qual o operador verificou a melhor opção entre todos os produtos cadastrados, obteve rendimento de $45,09 \%$ no sortimento "Serraria 1" e de 35,79\% no sortimento "Serraria 2", considerando o volume total das toras.

A produção de madeira serrada obteve maior rendimento no sortimento "Serraria 1" quando comparado ao rendimento no sortimento "Serraria 2" (25,98\%).

O melhor rendimento entre as simulações com otimização do desdobro foi o processamento considerando todas as opções de sortimento " $G$ ".

As simulações $E, D$ e $A$, as quais incluem a opção de produção de peças com uma polegada de espessura geram rendimentos que variam de 53,71 a 55,13\%, resultando em menor rendimento quando comparado a utilização de outras espessuras de produtos.

Entretanto, as simulações $\mathrm{F}$, B e $\mathrm{C}$ em que peças com espessura de duas ou três polegadas são priorizadas, produzem menor rendimento, variando de 36,81 a 49,00\%.

0 rendimento médio da madeira serrada ("Serraria 1 " e Serraria 2") na presente pesquisa foi de $40,44 \%$, superior ao encontrado por Fontes (1994) (30,5\%).

0 rendimento médio da madeira simulada foi de $50,22 \%$, superior ao encontrado por Ribas et al. (1989) $(48,5 \%)$, semelhante ao relatado por Olandoski et al. (1998) (50\%) e inferior aos valores encontrados Manhiça (2010) (52\%), Murara Junior et al. (2005) (53,6\%), Pinto et al. (2006) (57\%). Todavia, dentro do intervalo relatado por Cardoso Junior (2008) de 32,6 a 61,7\%.

\section{Análise econômica da madeira serrada}

O benefício econômico do desdobro e das simulações testadas, são apresentadas nas Tabelas 6 e 7.

Tabela 6. Benefício econômico para sortimento Serraria 1

\begin{tabular}{|c|c|c|c|c|}
\hline Simulação & $\begin{array}{c}\text { Matéria } \\
\text {-prima }\end{array}$ & $\begin{array}{c}\text { Receita } \\
\text { bruta }\end{array}$ & $\begin{array}{c}\text { Receita } \\
\text { parcial }\end{array}$ & $\begin{array}{c}\text { Benefício } \\
\text { econômico } \\
(\%)\end{array}$ \\
\hline MS & \multirow{8}{*}{ 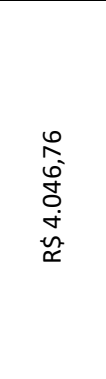 } & $\mathrm{R} \$ 8.620,16$ & $\mathrm{R} \$ 4.573,39$ & 113,01 \\
\hline A & & $\mathrm{R} \$ 10.557,36$ & $\mathrm{R} \$ 6.510,59$ & 160,88 \\
\hline B & & $\mathrm{R} \$ 7.902,46$ & $R \$ 3.855,69$ & 95,28 \\
\hline C & & $\mathrm{R} \$ 8.077,65$ & $\mathrm{R} \$ 4.030,88$ & 99,61 \\
\hline D & & $\mathrm{R} \$ 10.614,55$ & $\mathrm{R} \$ 6^{6.567,78}$ & 162,30 \\
\hline E & & $\mathrm{R} \$ 10.615,64$ & $R \$ 6.568,88$ & 162,32 \\
\hline $\mathbf{F}$ & & $R \$ 9.725,67$ & $\mathrm{R} \$ 5.678,90$ & 140,33 \\
\hline G & & $\mathrm{R} \$ 10.656,74$ & $\mathrm{R} \$ \mathbf{6}^{6.609,98}$ & 163,34 \\
\hline
\end{tabular}

O benefício econômico da madeira serrada no sortimento "Serraria 1" variou de $113,01 \%$ e no sortimento "Serraria 2" variou de 166,04\% e em ambos os sortimentos a simulação $G$ apresentou o maior benefício econômico (163,34 e 314,36\%).

As simulações $E, D$ e $A$ em que existe a opção de produção de peças com uma polegada de espessura geram benefícios econômicos melhores quando comparado as 
simulações $F$, B e C em que peças com espessura de duas ou três polegadas são priorizadas.

Tabela 7. Benefício econômico para sortimento Serraria 2

\begin{tabular}{|c|c|c|c|c|}
\hline Simulação & $\begin{array}{c}\text { Matéria- } \\
\text { prima }\end{array}$ & $\begin{array}{c}\text { Receita } \\
\text { bruta }\end{array}$ & $\begin{array}{c}\text { Receita } \\
\text { parcial }\end{array}$ & $\begin{array}{c}\text { Benefício } \\
\text { econômico } \\
\text { (\%) }\end{array}$ \\
\hline MS & \multirow{8}{*}{$\begin{array}{l}\hat{\infty} \\
\stackrel{1}{N} \\
\infty \\
-i \\
\tilde{n} \\
\sim\end{array}$} & $\mathrm{R} \$ 4.982,64$ & $\mathrm{R} \$ 3.109,77$ & 166,04 \\
\hline A & & $\mathrm{R} \$ 7.527,34$ & $\mathrm{R} \$ 5.654,47$ & 301,91 \\
\hline B & & $\mathrm{R} \$ 6.333,21$ & $\mathrm{R} \$ 4.460,35$ & 238,16 \\
\hline C & & $\mathrm{R} \$ 5.166,77$ & $R \$ 3.293,90$ & 175,87 \\
\hline D & & $\mathrm{R} \$ 7.729,33$ & $\mathrm{R} \$ 5.856,47$ & 312,70 \\
\hline E & & $\mathrm{R} \$ 7.756,38$ & $\mathrm{R} \$ 5.883,51$ & 314,14 \\
\hline $\mathbf{F}$ & & $\mathrm{R} \$ 6.854,80$ & $\mathrm{R} \$ 4.981,93$ & 266,01 \\
\hline G & & $\mathrm{R} \$ 7.760,36$ & $\mathrm{R} \$ \mathbf{5} .887,50$ & 314,36 \\
\hline
\end{tabular}

Analisando os resultados do benefício econômico das toras com sortimento "Serraria 1" e "Serraria 2", verificouse que atualmente o investimento em toras no sortimento "Serraria 2" proporcionam maiores margens às indústrias.

Em análise deste fato, verificou-se que o preço da madeira serrada produzida com toras de maior diâmetro é inferior quando comparado ao preço da madeira serrada produzida com toras finas.

Apesar das toras finas, não conseguirem produzir alguns tipos de produtos o mercado apresenta tendência ao seu uso.

Segundo Vital (2008), as serrarias de desdobro de toras do gênero pinus utilizam em sua grande maioria, sortimentos com diâmetros menores. Isto em função da redução dos diâmetros utilizados para a produção de compensados e consequente diminuição na demanda de toras para esse segmento.

Dobner Júnior et al. (2012) estudando o rendimento em serraria de toras de Pinus na região de Campo Belo do Sul, estado de Santa Catarina, verificaram que o abastecimento da indústria madeireira com toras de menores diâmetros, tem sido uma prática comum, remetendo a questionamentos tanto sobre o processo industrial de desdobro de toras como em relação à qualidade do produto final.

Os mesmos autores, verificaram que o benefício econômico é similar para toras nas classes de sortimento entre 20 e $25 \mathrm{~cm}$ e classes acima de $45 \mathrm{~cm}$ de diâmetro, entretanto para a classe de 25 a $45 \mathrm{~cm}$ o benefício custo é reduzido em aproximadamente $50 \%$.

\section{CONCLUSÕES}

O rendimento de madeira serrada de forma não programada apresentou grande variação quando comparado às classes de sortimento otimizadas.

As classes de sortimento com menores diâmetros apresentam maior benefício econômico, quando comparadas às classes de sortimento com maiores diâmetros considerando os valores atuais da matériaprima e da madeira serrada.

\section{AGRADECIMENTOS}

À Madsul Comercial Exportadora de Madeiras Ltda, pela autorização e apoio no trabalho de coleta dos dados.

\section{REFERÊNCIAS}

CARDOSO JUNIOR, A.A. Inovação tecnológica na obtenção de madeira serrada de pinus com uso de programa otimizador de desdobro. 2008. 120p. (Tese de doutorado).

DOBNER JÚNIOR, M. et al. Rendimento em serraria de toras de Pinus taeda: Sortimentos de grandes dimensões. Floresta e Ambiente, v.19, n.3, p.385-392, 2012.

DOBNER JÚNIOR, M. et al. Rendimento de laminação por faca de toras de Pinus taeda L. Scientia Forestalis, v.41, n.100, p.469-475, 2013.

FAGUNDES, H.A.V. Produção de madeira serrada e geração de resíduos do processamento de madeira de florestas plantadas no Rio Grande do Sul. 2003. 173p. (Dissertação de mestrado).

FONTES, P.J.P. Auto-suficiência energética em serraria de Pinus e aproveitamento dos resíduos. 1994. 140p. (Dissertação de mestrado).

HEINRICH, D. Simulação da produção de madeira serrada. 2010. 157p. (Dissertação de mestrado).

HOLLOCKS, B.W. Forty years of discrete-event simulations - a personal reflection. Journal of the Operational Research Sociaty, v.57, p.1299 -1883, 2006.

LEITE, H.G. Conversão de troncos em multiprodutos da madeira, utilizando programação dinâmica. 1994. 230p. (Tese de doutorado).

MADSUL. Lista de preços: Lista de produtos e grupo de produtos de madeira serrada de Pinus. 2015.

MANHIÇA, A.A. Rendimento e eficiência no desdobro de Pinus sp. utilizando modelos de corte numa serraria de pequeno porte. 2010. 85p. (Dissertação de mestrado).

MURARA JUNIOR, M.I. et al. Rendimento em madeira serrada de Pinus taeda para duas metodologias de desdobro. Floresta, v.35, n.3, p.473-483, 2005.

MURARA JUNIOR, M.I. et al. Estimativa do rendimento em madeira serrada de Pinus para duas metodologias de desdobro. Floresta e Ambiente, v.20, n.4, p.556-563, 2013. 
NOVAK, R.S. Um sistema de otimização aplicada ao desdobro de madeira. 2007. 126p. (Dissertação de mestrado).

OLANDOSKI, D.P. et al. Avaliação do rendimento em madeira serrada, qualidade e quantidade de resíduos no desdobro de Pinus spp. Ciências Agrárias, v.17, n.1-2, p.177-184, 1998.

OPTIMBER. Guia de instruções do usuário: MaxiTora - Software de otimização de desdobro de toras, da empresa OpTimber, ambiente Windows. 2016.

PINTO, I. et al. Simulatedvand realised industrial yields in sawing of maritime pine (Pinus pinaster Ait). HolzalsRoh- undvWerkstoff, v.64, p.30-36, 2006.

RIBAS, C. et al. Estudo da influência do diâmetro e do comprimento das toras de Pinus elliottii na produção de madeira serrada e de resíduos de serraria. Instituto Florestal, v.1, n.1, p.51-65, 1989.

ROCHA, M.P. Eucalyptus grandis Hill ex Maiden e Eucalyptus dunnii Maiden como fontes de matéria-prima para serrarias. 2000. 157p. (Tese de doutorado).

SILVA, J.C. Eucalipto a madeira do futuro. Madeira, v.11, n.59, p.4, 2001.

VITAL, B.R. Planejamento e operação de serrarias. Viçosa: UFV, 2008. 